\title{
Combination of tumor cell anti-adhesion and anti-tumor effect to prevent recurrence after cytoreductive surgery in a mice model
}

\author{
Johanne SEGUIN ${ }^{1}$, Cynthia PIMPIE ${ }^{2,3}$, Pétronille ROY ${ }^{24}$, Chantal AL SABBAGH ${ }^{1,4}$, Marc POCARD MD. \\ PhD. ${ }^{2,3}$, Nathalie MIGNET ${ }^{1} *$ PhD, Dr Vincent BOUDY ${ }^{1,4}$ Pharm.D. PhD. \\ 1-Université de Paris, UTCBS_Unité de Technologies Chimiques et Biologiques pour la Santé, CNRS, INSERM, 4 \\ avenue de l'observatoire, PARIS. nathalie.mignet@parisdescartes.fr; 2- Université de Paris, UMR 1275 : CAP \\ Paris Tech, Carcinomatosis Peritoneum Paris Technology ; 3- Hôpital Lariboisière 2 rue Ambroise Paré 75010 \\ Paris, France ; 4-Département Recherche et Développement Pharmaceutique, Agence Générale des \\ Equipements et Produits de Santé (AGEPS), AP-HP, 7 rue du fer à moulin, 75005 Paris, France.
}

\section{Novelty and Impact}

A tumor cell anti-adhesive thermogel was used as a drug delivery system to prevent tumor cells implantation in a peritoneal metastatic context. The bioavailability and the efficacy of the formulation was investigated and offered a first proof of concept in preclinical mice model.

\begin{abstract}
One of the main problems of colorectal cancer is not the treatment of the primary tumor but the metastatic stage. Means of metastatic spread is the invasion of the peritoneal cavity which leads to peritoneal metastasis (PM). PM cannot be easily cured, and the current treatments is rather heavy, combining cytoreductive surgery with intravenous and intraperitoneal chemotherapy. This therapeutic procedure is associated with significant morbidity, altered patient quality of life and poor prognosis. We postulated that development of a prophylactic treatment could be of high interest in this context. In this study, we formulated an anti-adhesive thermogel which contains chemotherapeutics to play a role of a barrier against tumor cells implantation, avoiding their adhesion and treating the remaining tumor cells with chemotherapy intraperitoneally in a mice model of PM. The bioavailability of the thermogel was tested intraperitoneally in mice. No sign of toxicity was observed in terms of change in body weight, anatomopathology and blood biomarkers. In vitro experiments proved that the thermogel induced limited adhesion of the tumor cells. Loading of oxaliplatin (Ox) and 5-Fluorouracil (5-FU) into the thermogel were able to significantly decreased peritoneal carcinomatosis index $(\mathrm{PCl})(-58 \%)$ and ascites $(-70 \%)$ in a murine model of peritoneal metastases. These pre-clinical results confirmed that smart thermogel associated with standard chemotherapy 5-FU and Ox could be a good candidate to decrease the risk of tumor cell implantation during cytoreductive surgery and prevent future metastatic process.
\end{abstract}

Keywords: Peritoneal carcinomatosis, thermogel, poloxamer, chemotherapy. 


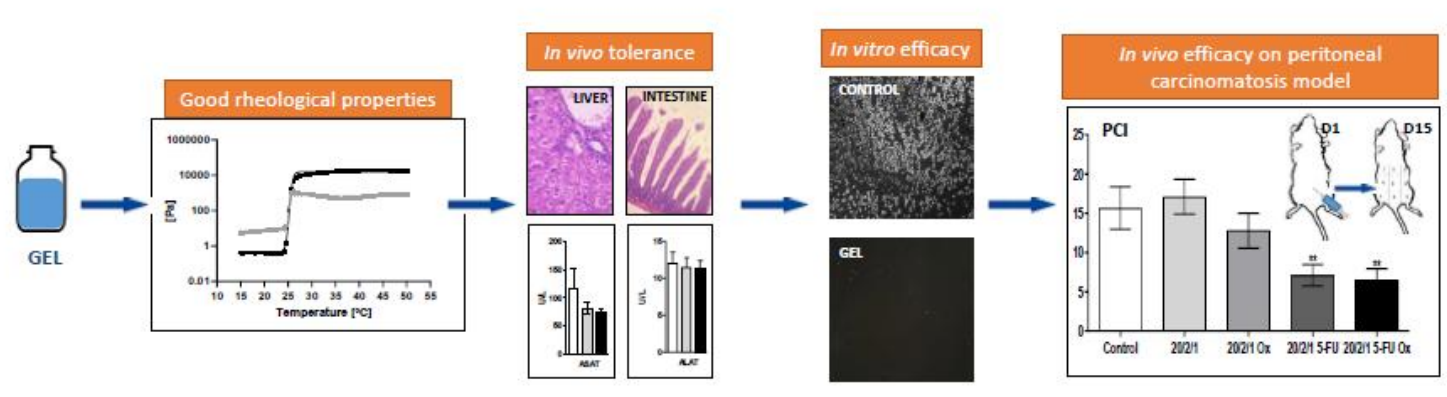

\section{Introduction:}

Peritoneal metastasis (PM) is considered as a terminal disease for a majority of etiology. Despite improvement in the overall outcome using recent therapeutic developments and aggressive treatment strategies with cytoreductive surgery followed by hyperthermic intraperitoneal chemotherapy (HIPEC), survival remains limited for colorectal cancer PM. A pooled analysis of North central cancer treatment group Phase III trials, reported a median overall survival (OS) at 12 months shorter than for other metastatic site ${ }^{1}$. During the last decade many efforts have been done to understand and anticipate the risk of PM process. For primary colon cancer, risk factors have been identified as advanced tumor $(\mathrm{T})$ status, perforated tumor, advanced node $(\mathrm{N})$ status, ovarian tumor location and limited carcinomatosis associated resection ${ }^{2-4}$. For gastric cancer, risk factors are more probably related to the surgical resection and advanced tumor status ${ }^{5}$. Free tumor cells could adhere post- surgery to form a metastatic mass. Prevention of tumor cells adhesion post-surgery seems obvious in this context. The difficulty is that the prophylactic treatment should be easily implemented according to the clinical situation found by the surgeon.

Prevention of PM have been proposed using different strategies. For some authors, a systematic second look could be proposed and prophylactic hyperthermic intraperitoneal chemotherapy (HIPEC) delivered ${ }^{6}$. However, a randomized trial testing that therapeutic solution has recently failed ${ }^{7}$. A clinical trial is actually ongoing for gastric cancer without PM giving prophylactic HIPEC at the end of the gastric resection ${ }^{8}$. The major limitation of that strategy is the fact that in case of success, common cases of gastric or colonic cancer surgery will have to be performed in highly specialized teams able to perform HIPEC. Regarding the cost and the health care organization, it is not realistic for many countries. As a result, an easy-to-use preventing treatment of PM is still needed.

PM is caused for a part by free cancer cells exfoliated from tumors invading the serosal layer. If surgeons are able to detect during the surgical resection that a high risk exist, it could be possible to give at that time a preventive drug administration using a drug delivery carrier containing chemotherapy. The advantage of that strategy is to offer a cost-effective solution to all surgeons 
without the need of a specific material. We, and others, have tested various chemotherapies and carriers to offer surgeons an easy to use solution to treat suspicious carcinomatosis ${ }^{9}$. We report here a new association of a specific gel carrier able to control tumor cell implantation, mimicking a clinical situation in an animal cancer orthotopic model.

Regarding these considerations, the pharmaceutical form was developed with a dual objective i) liquid at ambient temperature to have an easy form to handle in the surgery room ii) promote antiadhesion of tumor cells. Smart hydrogel with a sol-gel phase transition was chosen for these properties. Thermosensitive hydrogels present numerous advantages. The sol-phase of the formulation confers a homogeneous preparation and allows easy sterilization, administration and dose adjustment. The gel-state insures in situ gelification and consequently local and prolonged action ${ }^{10}$. A review of the various delivery systems proposed in this pathology was recently submitted by our group ${ }^{11}$. Based on this survey and the clinical requirements, the composition of the thermogel was chosen with the following objectives i) able to gelify around $27-30^{\circ} \mathrm{C}$ providing both a transferrable manufacturing process and facilitating local injection of the solution in the peritoneal cavity which will turn to a gel at body temperature ii) viscoelastic properties tending towards ideally elastic (tan $\delta$ lower than 0.1 ) iii) prevent tumor cell adhesion iv) able to carry and release a combination of cytotoxic agents v) involving no local or systemic toxicity for safety of use.

To answer these multiple objectives, we turned to already approved pharmacopeia excipients and based our formulation on poloxamer main content to provide the thermosensitive property. Poloxamer 407 (P407) is a triblock copolymer consisting of a central hydrophobic block of polypropylene glycol of 56 repeat units flanked by two hydrophilic blocks of polyethylene glycol (PEG) constituted by 101 repeat units. It is well known for its capacity to self-assemble into micelles, than organize in a thermogel in response to increasing concentration and/or temperature ${ }^{10}$. Interestingly, this polymer has been proposed in the literature to reduce post-operative adhesions after infertility surgery, laminectomy or laparoscopy ${ }^{12-14}$. Some authors also added copolymers in the formulation to reduce post-operative pelvic adhesions. Kim et al. showed that poloxamer/alginate better prevent pelvic adhesion severity and microscopic fibrosis as compared to hyaluronic acid/cellulose ${ }^{15}$. More recently, Cho et al. designed P407 nanodisks charged with rapamycin or paclitaxel to prevent post-surgical adhesions and reduce tumor burden in the peritoneal cavity ${ }^{16}$.

In this study, we proposed an original composition of poloxamers and alginate containing a combination of anticancer drugs clinically approved for colorectal cancer treatment 5-fluorouracile (5-FU) and oxaliplatin (Ox) to empeach tumor cell adhesion and dissemination during the surgery of colorectal carcinoma, and treat left inoperable metastases ${ }^{17}$. To answer this goal, mechanical resistance and rheofluidising properties of the thermogel were assessed. Compatibility and efficacy 
of the combination of drugs embedded within the gel were determined by HPLC and in vitro. The biological compatibility and the vivo efficacy were evaluated following the development of a PM development in a model of colorectal carcinomatosis in immune competent mice.

\section{Materials and methods}

\section{Materials}

The thermogels were prepared using sterile water Versylene ${ }^{\circledR}$ purchased from Fresenius Kabi France (Sèvres, France). Kolliphor ${ }^{\circledR}$ P407 and Kolliphor ${ }^{\circledR}$ P188 of pharmaceutical use grade were a kind gift from BASF (Ludwigshafen, Germany). 5-FU (Acofarma,) and Ox (Cipla Banglore,) were of pharmaceutical grade.

\section{Preparation of the thermogel}

The necessary amounts of P407 and P188 were mixed with approximately two thirds of the targeted final solution volume of water (Millipore, $0.22[\mathrm{im}$, Resistivity= $18.3 \mathrm{MH} / \mathrm{cm}$ ] in a glass beaker. The mixture was stirred, while lying in an ice bath, during several hours with the help of a magnetic stirrer and then left overnight at $4^{\circ} \mathrm{C}$. The mixture was dissolved in the upcoming day and the volume was adjusted with water to the exact desired volume (with the help of a graduated cylinder). Desired amounts of alginate (Manucol DH, FMC BioPolymer, 9005-38-3) and anticancer agents were then added to the liquid hydrogel. The gelling composition was mixed until all components were dissolved and subsequently stored at $4{ }^{\circ} \mathrm{C}$. Gel composition will be expressed in the following experimental section as: (w/v) \% P407/ (w/v) \% P188/ (w/v) \% alginate / (w/v) \% anticancer agents.

\section{Rheological characterization of thermogel}

The rheology measurements were carried out on rheometer (Anton Paar model MCR 102, Courtaboeuf, France), using a cone-plane geometry CP50-1 (50 mm; $\left.1^{\circ}\right)$ and an air gap of $0.10 \mathrm{~mm}$. The measurements were repeated 3 times with a volume of $750 \mu \mathrm{L}$ of thermogel. Non-destructive oscillatory measurements at $1 \mathrm{~Hz}$, was used to obtain the elastic modulus ( $\left.\mathrm{G}^{\prime}\right)$, the viscous modulus $\left(G^{\prime \prime}\right)$ and the phase angle $\left(\tan \sigma=G^{\prime \prime} / G^{\prime}\right)$. The data was then processed using the associated software Anton Paar Rheo Compass 1.20.

\section{Evaluation of $5-\mathrm{FU}$ and $\mathrm{Ox}$ compatibility at $4^{\circ} \mathrm{C}$ in the thermogel}

5-FU and Ox solution at the respective concentrations of $0.5 \% \mathrm{w} / \mathrm{v}(5 \mathrm{mg} / \mathrm{mL})$ and $0.1 \% \mathrm{w} / \mathrm{v}$ $(1 \mathrm{mg} / \mathrm{mL})$ were prepared separately or in combination in water for injection (WFI). The equivalent concentration of the drugs was prepared in P407/P188/alginate 20/2/1 \% thermogel. Aliquots of this 
preparation $(400 \mu \mathrm{L})$ were prepared and incubated at $4^{\circ} \mathrm{C}$ for the experiment. At 0,3 and 24 hours, a sample of $20 \mu$ Lwas drawn off and diluted 700 times then filtered with an Amicon 3 KDa filter (Amicon Ultra-0.5 ml, Ultracel ${ }^{\circledR} 3 \mathrm{Kda}$ ). The sample was then centrifuged at $14,000 \mathrm{rpm}$ for $40 \mathrm{~min}$. The filtrate obtained was analyzed by HPLC to determine the concentration of each drug using a Shimadzu LC- 20AD system (Duisburg, Germany) connected to a C18 column ( $\mu$ Bondapak C18, $10 \mu \mathrm{m}$, $125 \mathrm{~A}, 3,9 \times 300 \mathrm{~mm}$ (Waters WAT027324). Analyses were performed at $25^{\circ} \mathrm{C}$ with detection at 266 $\mathrm{nm}$ and $250 \mathrm{~nm}$ (fluorescence detector, RF-10AXL, Shimadzu). Analyses were performed on 3 separate samples.

Release studies

The hydrogel $(1 \mathrm{~mL})$ was introduced into a $20 \mathrm{~mL}$ vial and placed at $5^{\circ} \mathrm{C} \pm 3^{\circ} \mathrm{C}$ for $10 \mathrm{~min}$ in order to homogenize the distribution of the hydrogel at SOL state in the bottom of the vial. Afterwards, the vial was placed in an incubator set at $37^{\circ} \mathrm{C}$ and allowed to gelify during $30 \mathrm{~min}$. Subsequently, $5 \mathrm{~mL}$ of pre-warmed $\left(37^{\circ} \mathrm{C}\right)$ release medium $(\mathrm{PBS}, \mathrm{pH} 7.3,0.01 \mathrm{M})$, were gently layered over the surface of the gel and vials were stored in an orbital shaker/incubator (Orbital Shaker, Forma Scientific, Ohio, USA) and shaken at $50 \mathrm{rpm}$ at $37^{\circ} \mathrm{C}$. At specific time points $(0,0.25,0.5,1,2,24,48,96,168$ and 216 hours), the release medium (200 $\mu \mathrm{L})$ was sampled and replaced with an equal volume of prewarmed fresh PBS. The amounts of released 5 -fluorouracil and oxaliplatin were measured by HPLC, following the protocols described in the section above. All measurements were conducted in triplicate and mean values \pm standard deviations were reported.

\section{Cell line}

CT26-Luc cell line, a murine colon cancer, kindly provided by Prof. Lea Eisembach Weizmann (Institute of Science Rehovot, Israel) were grown in Dulbecco's Modified Eagle Medium (DMEM, Gibco) supplemented with $10 \%$ of fetal bovine serum (Gibco Life technologies) and $1 \%$ of antibiotic (100 $\mu \mathrm{M}$ streptomycin, 100U/mL penicillin, Gibco Life technologies). These cell lines were cultured at $37^{\circ} \mathrm{C}$ in a $5 \% \mathrm{CO}_{2}$-humidified atmosphere.

\section{Anti-adhesive effect of the thermogel}

6 well plate was coated with $300 \mu \mathrm{L}$ of the thermogel and incubated for $30 \mathrm{~min}$ at $37^{\circ} \mathrm{C}$. Then, 60.000 CT26-luc cells were seeded in each well. At 10, 20, 30, 45, 60, $90 \& 120$ minutes the cell monolayer was washed, fixed with $75 \%$ of ethanol and finally stained with haematoxylin eosin. The adherent cells were then counted as comparison with control wells without thermogel. The evaluation of the adhesion was realized on 8 pictures and the result was expressed by mean \pm SEM. 
In vitro efficacy of combined Ox and 5-FU in the thermogel

Cells were seeded in Falcon ${ }^{\text {TM }}$ Cell Culture Inserts (Cyclopore ${ }^{\circledR}$ Falcon ${ }^{\circledR} 353095$ ) for $24 \mathrm{~h}$ at $37^{\circ} \mathrm{C}$ (800 000 cells per insert in $1 \mathrm{~mL}$ of medium). At day 2, the apical medium was removed and $200 \mu \mathrm{l}$ of thermogel or culture medium control solution was put on the insert. At day 3, plates were incubated at $4^{\circ} \mathrm{C}$ for ten minutes, in order to liquefy the gel and the wells were wash two times with culture medium, to removed dead cells and thermogel residues. A solution of the 3-(4,5- Dimethylthiazol-2yl)-2,5-diphenyltetrazolium bromide (MTT, Sigma Aldrich, 298-93-1; $0.5 \mathrm{mg} / \mathrm{mL}$ ) in culture medium was incubated with cells for 4 hours. The solution was removed and replaced by $100 \mu \mathrm{L}$ of Dimethyl sulfoxide DMSO and the plate was shaken during $10 \mathrm{~min}$. The absorption was subsequently measured at $562 \mathrm{~nm}$ (TECAN Infinite F200 Pro, Tecan Trading AG, Switzerland) in a 96-well plate (TPP Techno Plastic Products, 92024). The viability was estimated in percent of absorbance of the control wells.

In vivo tolerance of the thermogel

In vivo experiments were performed with respect to standard European-community-guideline requirements for laboratory-animal care and use. The laboratory number of national accreditation for animal experimentation is C75-10-03 (November 27-2012). The registration number of the ethics committee for the experiments is APAFIS = 3944-2015 $122813591563 \mathrm{v} 3$, the project is authorized as number 02095-03 at the date of March 25 - 2016 for a 5 years period. For the experimentation, only female mice BALB/C (Charles River, Arbresle, France) were used because of difference in tumor growth as compared to male mice. Female BALB/cJRj mice were injected with $250 \mu \mathrm{L}$ of P407 $20 \%$ thermogel $(n=8)$ or P407/P188/alginate $20 / 2 / 1 \%$ thermogel $(n=8)$ in the peritoneal cavity. The weight monitoring was realized every 2-3 days. 14 days after thermogel injection, mice were anesthetized (isoflurane $2 \%$ ) and the blood was collected with cardiac puncture for the evaluation of hemotoxicity and hepatic toxicity. Then, mice were sacrificed by cervical dislocation and brain, liver, kidney, lung and intestine were removed and were fixed in phosphate buffer $4 \%$ formaldehyde for anatomopathology. The organs were included in paraffin, cut and the routine diagnostic pathology hematoxylin and eosin (HE) stain was applied onto the organ slices obtained.

\section{Efficacy of combined Ox and 5-FU thermogel in peritoneal carcinomatosis model}

A murine model of peritoneal carcinomatosis was obtained through an intraperitoneal injection (IP) of $1 \times 10^{4}$ CT26-Luc cells in order to mimic the spelling of tumor cells that can happens during a surgical procedure. Different treatments were injected $1 \mathrm{~h}$ after injection of tumor cells, and five different groups of mice $(n=10)$ were studied: (i) control group: water for injection, (ii) P407/P188/alginate in 20/2/1\%, (iii) P407/P188/alginate/5-FU 20/2/1/0,24\% w/v (5-FU 2,4 mg /mL), 
(iv) P407/P188/alginate/Ox 20/2/1/0,048 \% w/v (Ox 0,48 mg /mL) (v) P407/P188/alginate/5-FU/Ox $20 / 2 / 1 / 0,24 / 0,048 \% \mathrm{w} / \mathrm{v}$. The well-being of mice was checked twice a week. At day 15, mice were killed by cervical dislocation. Then, a postmortem examination was performed through a laparotomy. Visceral and parietal peritoneum were analyzed to detect nodules and the peritoneal carcinomatosis index $(\mathrm{PCl})$ was calculated according as previously described ${ }^{18}$ Briefly, the abdomen was divided in 12 zones and a scoring was established from 0 to 3 according to the presence and the size of tumor nodules.

\section{Statistical analysis}

Analysis was performed with GraphPad Prism software. A Spearman test was applied to evaluate the correlation between shear modulus and viscosity for the 20/2/1 formulation. The 2 ways ANOVA with Bonferroni's multiple comparisons test was used for the comparison of the weight after i.p. injection of the formulation. A nonlinear fit exponential growth equation was applied on the adhesion result and Sidak's multiple comparisons statistical analysis was realized. The Cytotoxicity of the chemotherapeutic agents 5-FU and Ox and the in vivo efficacy was assessed with one way ANOVA with turkey multiple comparison. For all of the result the statistical significance was resumed as follows: Ns $\mathrm{P}>0.05{ }^{*} \mathrm{P} \leq 0.05, * * \mathrm{P} \leq 0.01, * * * \mathrm{P} \leq 0.001, * * * * \mathrm{P} \leq 0.0001$

\section{Results}

\section{Rheological characterization of the thermogel}

In order to determine the best formulation corresponding to the target profile, we performed rheology experiments at $37^{\circ} \mathrm{C}$. The results obtained are listed in Figure 1 . The measurements were carried out in a linear regime to be free from the action of the deformation and to focus only on the effect of temperature on the studied thermogel. The gelation temperature (Tgel) was determined by measuring the elastic $\left(G^{\prime}\right)$ and viscous $\left(G^{\prime \prime}\right)$ moduli of the thermogel as a function of the temperature. The crossing of the moduli indicates the sol-gel phase transition and therefore the Tgel (Figure 1A-B). The Tgel was comprised between 25.4 and $27.7^{\circ} \mathrm{C}$, meaning an injectability of all formulations tested at surgery room temperature. If we consider the elasticity value, the targeted criterion was respected and was found upper than $10000 \mathrm{~Pa}$. For further experiments, we chose the combination with the higher $G^{\prime}$ value $(20 / 2$ and 20/2/1). An example of the rheological curve obtained for these 2 formulations are represented in Figure 1AErreur ! Source du renvoi introuvable..

The ratio between loss and storage moduli (Tanס) indicate the loss factor (Figure 1B). With a loss factor near 0 , thermogel is assimilated to viscoelastic solid. This shows an internal mechanical 
resistance towards external mechanical constraints despite the absence of gel reticulation. The loss factor obtained for this gel therefore allows to predict a good mechanical resistance at $37^{\circ} \mathrm{C}$ after in situ administration.

The inverse correlation between the viscosity and the Shear modulus indicate a pseudoplastic behavior of the formulation P407/P188/alginate 20/2/1 (Figure 1C). This property is crucial for the injectability of the thermogel, but also for a good covering of the peritoneal cavity.

A

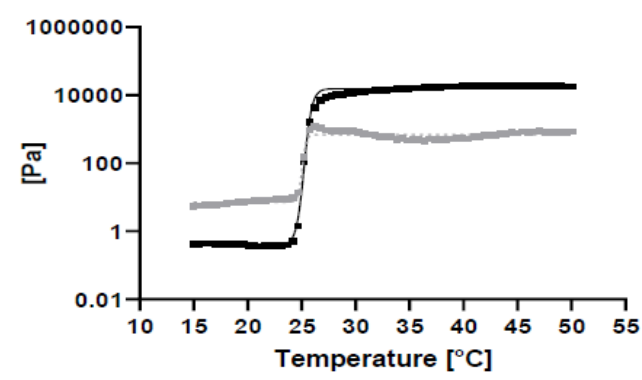

B

\begin{tabular}{|l|l|l|l|l|}
\hline $\begin{array}{l}\text { P407 / P188 / Protanal } \\
\% w t\end{array}$ & Tgel $\left({ }^{\circ} \mathrm{C}\right)$ & $\begin{array}{l}\mathrm{G}^{\prime}(\mathrm{Pa}) \\
37^{\circ} \mathrm{C}\end{array}$ & $\begin{array}{l}\mathrm{G}^{\prime \prime}(\mathrm{Pa}) \\
37^{\circ} \mathrm{C}\end{array}$ & $\begin{array}{l}\text { Tano } \\
37^{\circ} \mathrm{C}\end{array}$ \\
\hline $20 / 2 / 0$ & $26.0 \pm 0.1$ & $18200 \pm 500$ & $590 \pm 60$ & $0.03 \pm 0.002$ \\
\hline $20 / 2 / 0.25$ & $27.0 \pm 0.2$ & $14500 \pm 950$ & $620 \pm 90$ & $0.04 \pm 0.01$ \\
\hline $20 / 2 / 0.5$ & $26.0 \pm 0.1$ & $15200 \pm 100$ & $440 \pm 03$ & $0.03 \pm 0.000$ \\
\hline $20 / 2 / 1$ & $25.4 \pm 0.1$ & $17400 \pm 250$ & $500 \pm 09$ & $0.03 \pm 0.001$ \\
\hline
\end{tabular}

C

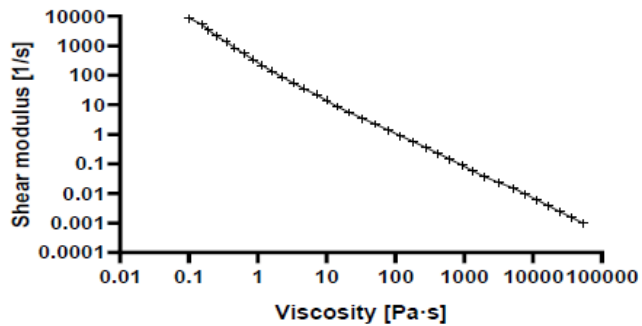

Figure 1: P407/P188/alginate thermogel rheological results. (A) Representation of the sigmoidal fitting curve of the viscous modulus G" (grey) and the elastic modulus G' (black) for 20/2/1 thermogel. (B) Rheological parameters of the different formulations tested. $n=3$ mean $\pm S D$. (C) Spearman correlation between shear modulus and viscosity for the P407/P188/alginate 20/2/1 formulation ( $\left.n=6, \mathrm{P}<0,0001^{* * * *}\right)$.

\section{In vivo tolerance of the main component of the thermogel}

The in vivo toxicity of the thermogel was first investigated. P407, main component in gel $(20 \% \mathrm{w} / \mathrm{v})$, and complete gel formulation P407/P188/alginate (20/2/1) were injected in mice intraperitoneally. The weight of the mice was followed for 14 days (Erreur ! Source du renvoi introuvable.). Despite the slight decrease of the weight (Erreur! Source du renvoi introuvable.A), observed for the complete formulation 20/2/1 $48 \mathrm{~h}$ after the injection (-2.57\%); no significant difference in weight variations was denoted between each group (Erreur ! Source du renvoi introuvable.B). 
Fourteen days after the injection described above, several toxicities analyses were undertaken (Figure 2C-M). Regarding white blood cells, lymphocytes, monocytes, granulocytes, platelets, red blood cells and hematocrit levels, no significant difference in blood analysis could be denoted (Figure 2C-E). Similarly, no significant difference was observed in transaminases dosage between each group which is in favor of good hepatic tolerance (Figure $2 \mathrm{~F}-\mathrm{G}$ ). Moreover, as we also expected, no toxicity was denoted in cerebellum, brain, kidney, small intestine, lung and liver anatomopatological slices (Figure $2 \mathrm{H}-\mathrm{M}$ ).

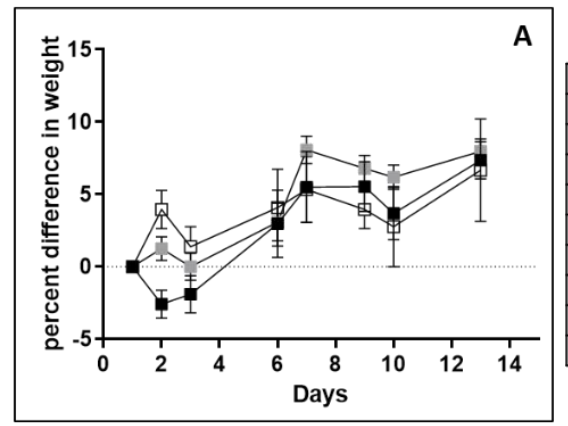

\begin{tabular}{|c|c|c|c|c|c|c|c|c|c|}
\hline B \\
\hline Days & \multicolumn{3}{|c|}{ Control } & \multicolumn{3}{|c|}{ P407 } & \multicolumn{2}{c|}{ P407/P188/Alginate 20/2/1 } \\
\hline & Mean & SEM & N & Mean & SEM & N & Mean & SEM & N \\
\hline 1 & 0 & 0 & 4 & 0 & 0 & 8 & 0 & 0 & 8 \\
\hline 2 & 3,95 & 1,32 & 4 & 1,25 & 0,82 & 8 & $-2,57$ & 0,98 & 8 \\
\hline 3 & 1,39 & 1,39 & 4 & 0,00 & 0,94 & 8 & $-1,88$ & 1,29 & 8 \\
\hline 6 & 4,09 & 2,65 & 4 & 3,10 & 1,31 & 8 & 2,97 & 2,31 & 8 \\
\hline 7 & 5,34 & 2,27 & 4 & 8,07 & 0,95 & 8 & 5,51 & 2,45 & 8 \\
\hline 9 & 3,95 & 1,32 & 4 & 6,79 & 0,90 & 8 & 5,55 & 1,71 & 8 \\
\hline 10 & 2,78 & 2,78 & 4 & 6,19 & 0,83 & 8 & 3,70 & 1,81 & 8 \\
\hline 13 & 6,67 & 3,54 & 4 & 7,98 & 0,85 & 8 & 7,36 & 1,29 & 8 \\
\hline
\end{tabular}
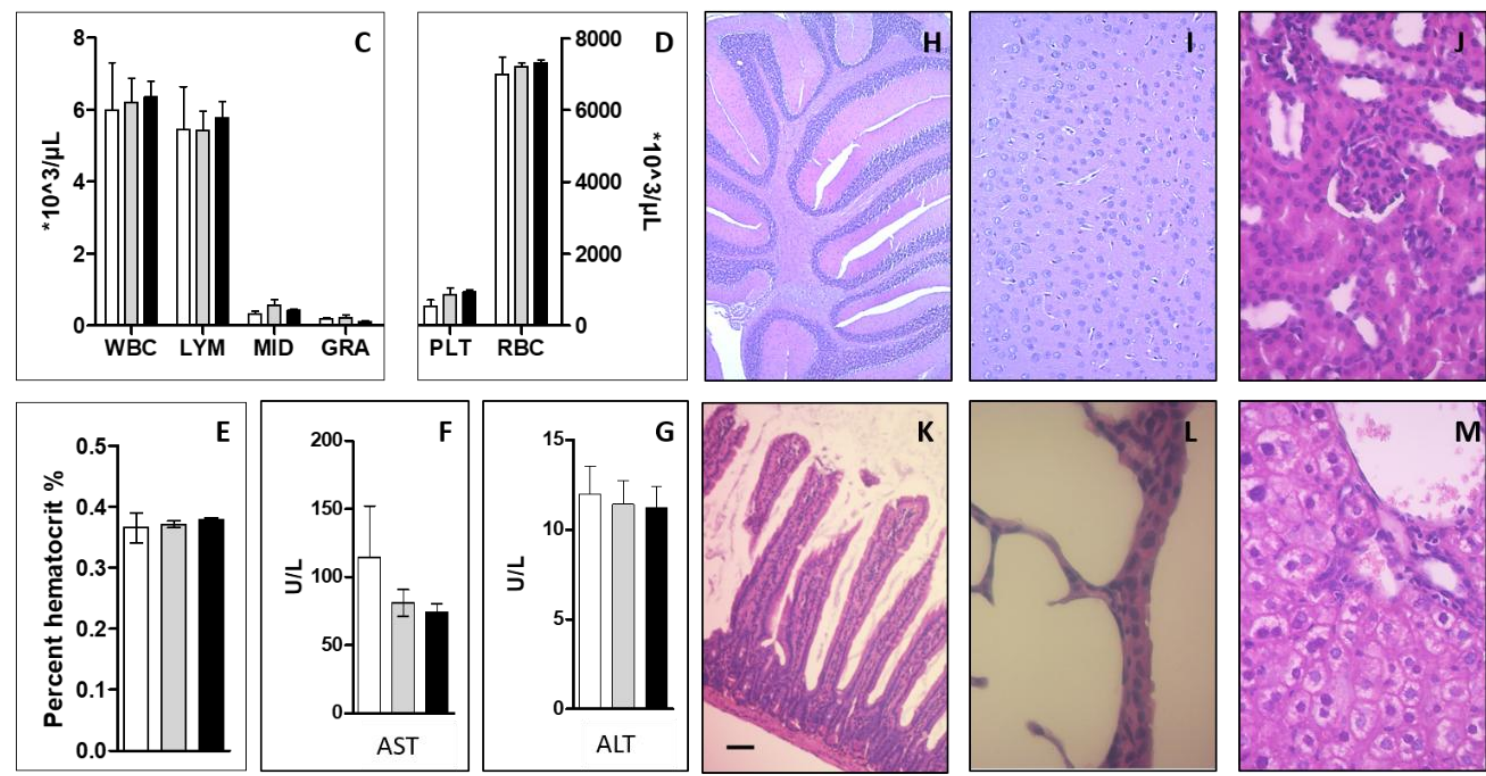

Figure 2

Figure 2: in vivo evaluation of the toxicity of the formulation in healthy mice $(n=8)$. Longitudinal monitoring of the weight after injection of the different formulation. (A) Percentage difference in weight following 14 days of the control (white), P407 20\% (grey) and P407/P188/ protanal 20/2/1 (black) groups. (B) corresponding value of the percentage difference in weight for each groups (100*value -baseline, mean,SEM and number of value). 2-way ANOVA with Bonferroni's multiple comparisons test: ns. (C, D, E) Effect of P407 20\% (grey) and the thermogel 20/2/1 (black) as function of control (white) on blood component and hematocrit level where WBC: white blood cell, LYM: lymphocytes; MID: Monocytes; GRA: Granulocytes; PLT: platelet; RBC: RED blood cell. Effect of P407 (grey) and the thermogel 20/2/1 (black) as function of control (white) on hepatic enzyme AST 
Aspartate Aminotransferase (F) ALT alanine transaminase (G). Effect of the key component of hydrogel: P407 on main organ, Hematoxylin eosin staining of cerebellum $(\mathrm{H})$, Brain (I), kidney $(\mathrm{J})$, small intestine (K), Lung (L) and Liver (M). Scale bar for images $\mathrm{H}$ to $\mathrm{M}$ corresponds to $50 \mu \mathrm{m}$.

In vitro anti-adhesive effect of thermogel on colon carcinoma cell line

After in vivo safety verification of the thermogel, its anti-adhesive effect was investigated. As shown in the pictures (Figure $3 \mathrm{~A}$ ) and in the resulting image quantification (Figure 3B), when the plate was coated with thermogel, cell adhesion was drastically reduced as compared to the control group after 90 and $120 \min (p<0,05$ and $p<0,001$, respectively).
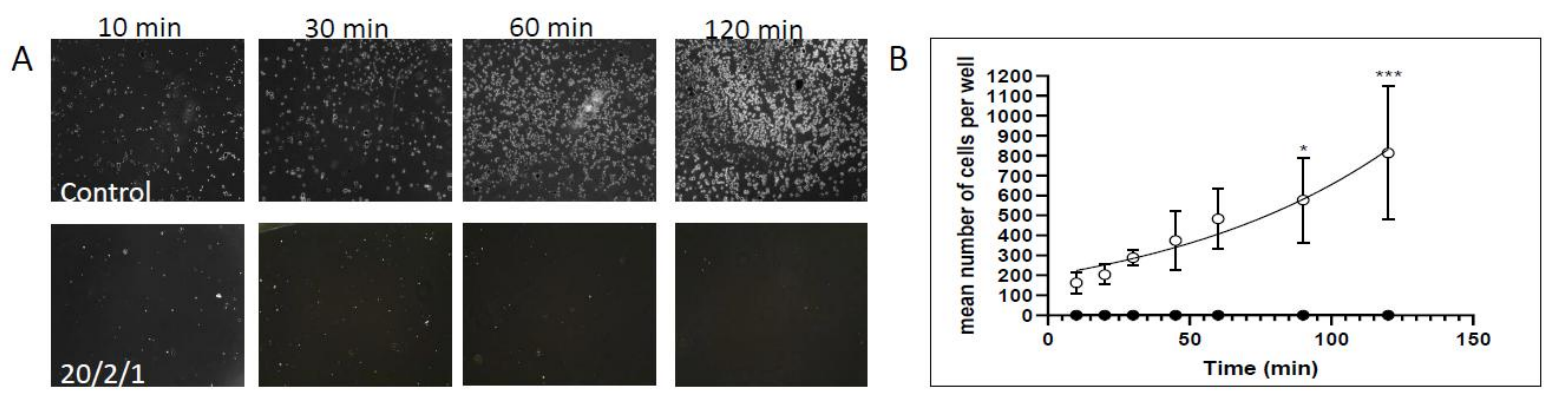

Figure 3: (A) Representative picture of CT26 cells incubated without (up) and with (down) 20/2/1 thermogel. (B) Mean cells counting in wells from 10 to 120 min after incubation with thermogel 20/2/1 (black dot) at $37^{\circ} \mathrm{C}$ or in control (white circle). A nonlinear fit exponential growth equation was applied on the result expressed in mean \pm SEM and Sidak's multiple comparisons statistical analysis was realized $p<0.05 * ; p<0.001 * * *$.

\section{Drug stability in thermogel}

In order to combine the therapeutic outcome of our thermogel, cytotoxic agents were solubilized within the polymers network. The first step was to verify the absence of interactions between the drugs and the thermogel. By comparing retention times of drugs in the admixture 5-FU/Ox with those of 5-FU and Ox solutions (Figure 4A-B), no modification was noticed. 5-FU was eluted at 9.5 $\min$ and $\mathrm{Ox}$ at $20.5 \mathrm{~min}$ (Figure $4 \mathrm{C}$ ).

5-FU and Ox concentrations were measured at 3 and 24 hours, in solution and thermogel. 5-FU in solution was stable over 24 hours. Ox in solution showed a steady concentration up to 3 hours postpreparation. Its concentration decreased then by $11 \%$ over 24 hours (Figure 4D). 5-FU incorporation into the thermogel had a very slight effect on its concentration. Ox concentration remained invariable up to 3 hours, though it decreased by $14 \%$ after 24 hours. The study of 5-Fluorouracil and oxaliplatin release from P407/P188/alginate/5-fluorouracil/oxaliplatin 20/2/1/0.5/0.1\% w/v thermogel was conducted in vitro at $37^{\circ} \mathrm{C}$. The release of the drugs from the thermogel was studied 
over time in PBS (pH 7.3;0.01 M). The data indicate a release of both Ox and 5-FU over $5 \mathrm{~h}$ before reaching a plateau and decreasing due to degradation in particular of Ox, known to be unstable at this $\mathrm{pH}$ (Figure 4E).
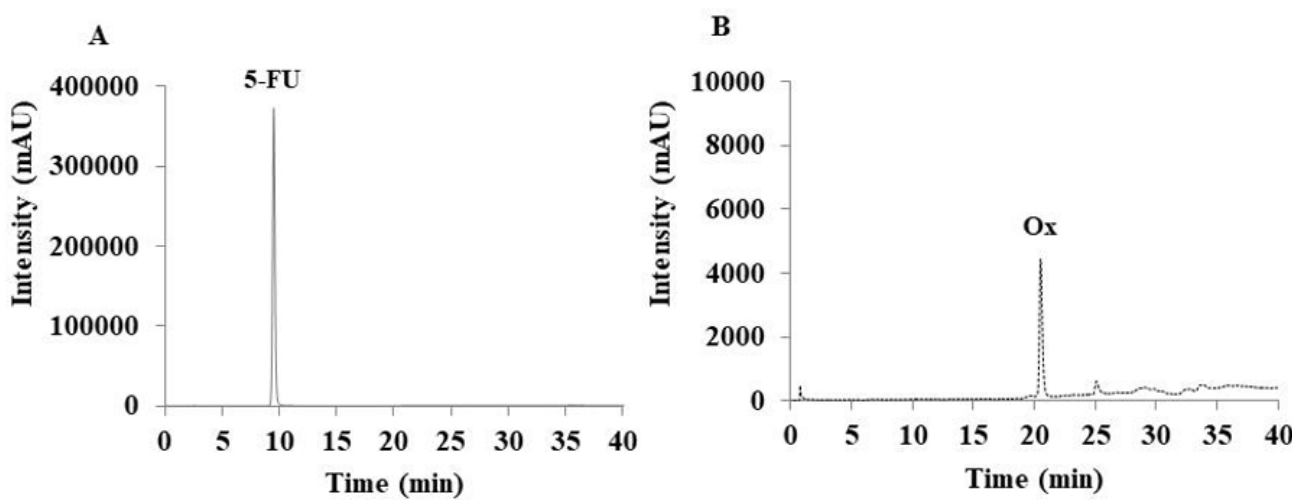

\section{C}
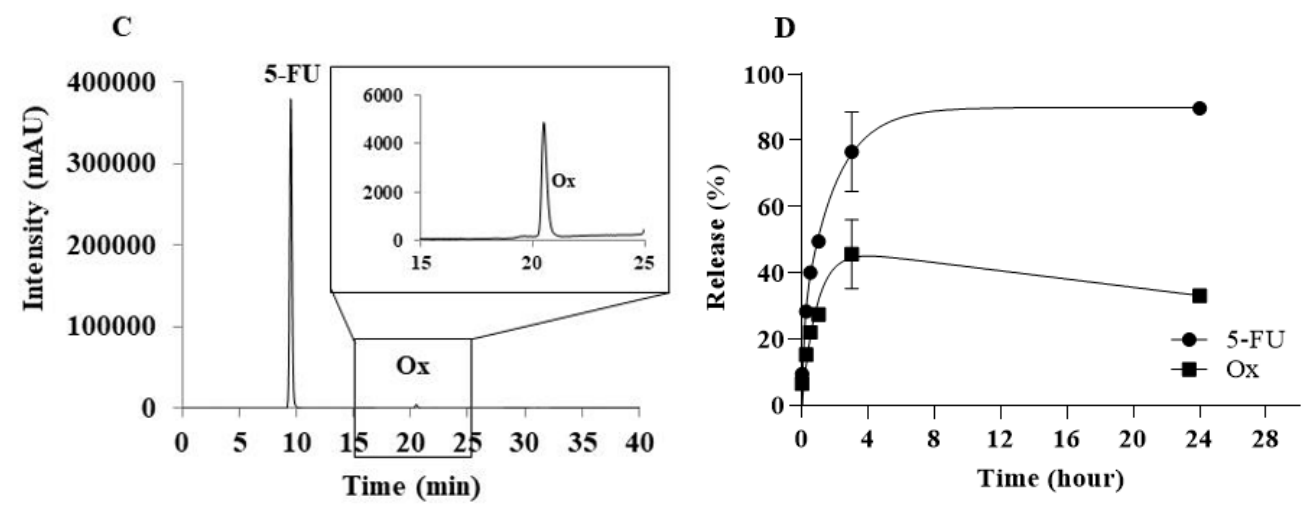

Figure 4: HPLC chromatograms of (A) 5-FU WFI, (B) Ox WFI and (C) 5-FU/Ox admixture at $266 \mathrm{~nm}$ (maximal wavelength of 5-FU). (D) determination of 5 - $\mathrm{FU}$ and $\mathrm{Ox}$ concentrations in 5 - $\mathrm{FU} / \mathrm{Ox}$ admixture, prepared in WFI or thermogel, over time at $4^{\circ} \mathrm{C}(\mathrm{n}=3)$.

In vitro efficacy of combined Ox and 5-FU in the thermogel

In order to validate the effect of the chemotherapeutic agent encapsulated in the 20/2/1 thermogel, the cytotoxicity of this formulation was investigated on CT26-luc cell line as compared to the chemotherapy at the same concentration in solution. The ratio was chosen based on 5-FU solubility in water at $20^{\circ} \mathrm{C}$. The final drug loading content corresponded to $0.5 \% \mathrm{w} / \mathrm{v}(5 \mathrm{mg} / \mathrm{mL})$ and $0.1 \% \mathrm{w} / \mathrm{v}$ $(1 \mathrm{mg} / \mathrm{mL})$ for 5-FU and Ox, respectively. As represented in Figure 5, cytotoxicity of 5-FU and Ox were maintained in thermogel and were significant as regard to the respective control $(* * *, p<0.0001)$. Moreover, no significant difference was observed between the solution and the thermogel with chemotherapeutic agent $(p=0.6981)$. On the other hand, thermogel without drugs had no impact on cell viability. 

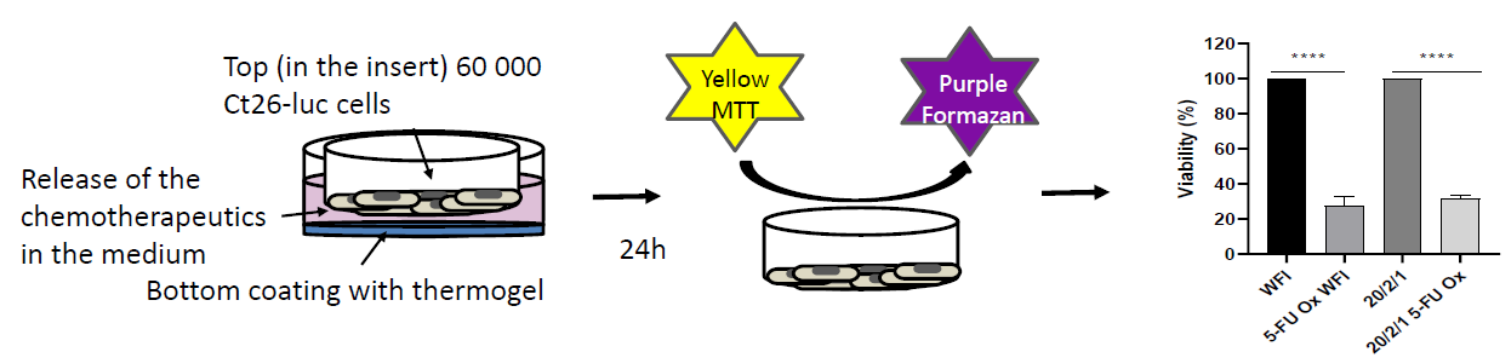

Figure 5: Cytotoxicity of the chemotherapeutic agents 5-FU and Ox embedded in the thermogel $24 \mathrm{~h}$ after incubation against CT26-luc colon carcinoma cell line. One way ANOVA with turkey multiple comparison $* * * * \mathrm{P}<0.0001$.

\section{Efficacy of combined Ox and 5-FU thermogel in peritoneal metastasis model}

Based on the in vitro efficacy of Ox and 5-FU entrapped in thermogel, in vivo assay on peritoneal metastasis model was undertaken. The murine model was obtained by injection of $1 \times 10^{4}$ CT26-Luc cells in the intraperitoneal region as described ${ }^{9}$. The efficacy evaluation of thermogel was based on the peritoneal carcinomatosis index $(\mathrm{PCl})^{19}$. Guided on the schedule shown on Figure $6 \mathrm{~A}$, formulations were injected $1 \mathrm{~h}$ post cell injection to better mimic the surgical situation. As can be seen in Figure 6B, formulations were well tolerated by the mice with non-significant change in body weight until day 3 then normalizing in the following days as compared to control group. As shown in Figure 6C-D, the empty thermogel had no effect both on the $\mathrm{PCl}$ and the number of areas involved. Interestingly, 5-FU combined or not with $\mathrm{Ox}$ within the thermogel reduced significantly the $\mathrm{PCl}$ as compared to control group $(p<0.005)$. However, only the combination of both $5-\mathrm{FU}$ and $\mathrm{Ox}$ within the thermogel was able to reduce the number of areas involved in peritoneal cavity $(p<0.05)$ and the number of mice with ascites (Figure 6D-E). Ascites volume was dramatically reduced using thermogel with Ox alone or combined with 5-FU (respectively $p<0.001$ and $p<0.005$ ) (Figure 6F). 

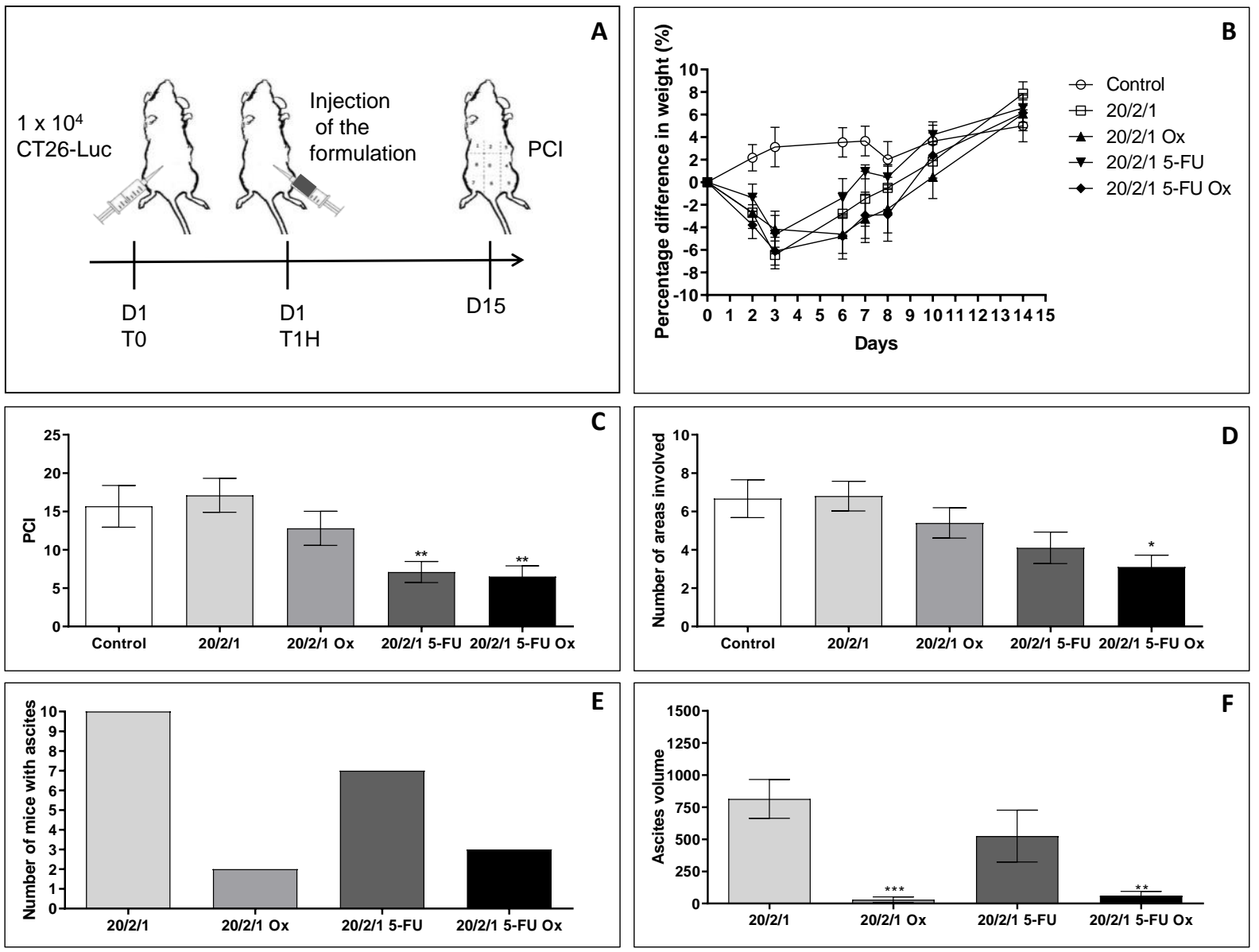

Figure 6: Evaluation of thermogel formulations efficacy on colorectal CT26-Luc peritoneal carcinomatosis model. Schedule of the experiment (A). Percentage difference in weight as function of time after formulations injection (B). PCl at day 15 after CT26-Luc cells induced carcinomatosis for the different formulations of thermogel (C). Number of areas involved at day 15 after CT26-Luc cells induced carcinomatosis for the different formulations of hydrogel (D). Number of mice with ascites (E) and mean ascites volume (F). ${ }^{*} p<0.05,{ }^{* *} p<0.005,{ }^{* * *} p<0.001$ as compared to control group (mean \pm SEM, $n=9-10$ ).

\section{Discussion}

Surgical resection of colon, ovarian or stomach cancers represents a high risk of PM or recurrence. Detachment of cancers cells during surgical procedure can lead to their implantation in peritoneal cavity where they proliferate to form new nodules scattered ${ }^{5,20,21}$. There is an important challenge to develop new drug delivery systems allowing sustained release associated with an anti-adhesive effect against of tumor cell implantation. Indeed, it is common when the surgeon operates that free cells are already present in the peritoneum. Moreover, when performing surgery, the surgeon also offers the proper environment for tumor implantation with the surgical scares. Fibrine is activated and fibrinogen allows tumor cell implantation during or just after surgery. These are the cells that we are targeting here using a local hydrogel just after the surgery. Actually, many surgical teams associated 
after cytoreductive surgery (CRS) an intraperitoneal treatment as chemotherapy with hyperthermia (HIPEC), or postoperative intra peritoneal drug delivery using direct and repeated injections. Our objective is to offer an esay to handle drug delivery systems for all surgeons which could be used in the surgery room according to the clinical situation encountered, in particular in case of high risk of secondary peritoneal spread after surgery.

In this context, we proposed an hydrogel based on a FDA approved excipient P407. P407 has the capacity to be in solution state at low temperatures, allowing easy injectability, usability, homogeneity and sterilizability, but also turning into gel state when temperature increases. The hydrogel does not need to stay for a long time in the peritoneal cavity, neither deliver drugs for a long period, the objective is to have a sufficiently lasting gel to prevent cell adhesion which occurs within two days post-surgery,

Physico-chemical properties of poloxamers can be improved by the addition of copolymers as referred by Qiu et al. ${ }^{22}$ which is why the composition of poloxamers was complexified in this study. Here we chose a mixture of two poloxamers P407 and P188, in a proportion leading to tunable solgel transition around $27-30^{\circ} \mathrm{C}^{23}$ in combination with alginate, a gelling agent which do not cause cell adhesion ${ }^{24}$. Previously, it had been shown that P188 reduced the adhesion rate of erythrocytes to endothelial cells when added to a Dextran $500 \mathrm{kDa}$ solution, which is a polymer known to induce cellular interaction and adhesion ${ }^{25}$. Steinleitner et al. also highlighted P407 ability to prevent postsurgical adhesion formation in hamster and rabbit uterine-horn injury models ${ }^{12}$. Those results are consistent with our in vitro significant findings of CT-26 cells adhesion decrease observed in presence of thermogel. Several studies also demonstrated the efficacy of alginate, in the prevention of postsurgical adhesion. Cho et al. tested various types of alginate (viscous solution, gel and film) and compared their anti-adhesion efficacy onto peritoneal surgical defect in comparison with Interceed ${ }^{\circledR}$, a commercialized cellulose-based adhesion barrier. Alginate gel and un-crosslinked films demonstrated a higher anti-adhesion power with a similar toxicity profile as compared to control and Interceed ${ }^{\circledR}$ groups $^{26}$. Chaturvedi et al. demonstrated also efficacy of an ultrapure alginate gel, in adhesion prevention in a rat model with caecal abrasion and peritoneal side wall excision. They did not find any difference in comparison with Seprafilm ${ }^{\circledR}$, a sodium hyaluronate carboxymethyl cellulose based membrane which was the most effective adhesion barrier previously reported ${ }^{27}$. Moreover, authors proved the superiority of their ultrapure alginate gel in preventing adhesion formation on a compromised anastomotic healing rat model. These results are in favor of an antiadhesive effect of the alginate which we took advantage of to propose a mixture of poloxamer 
containing alginate to further enhance the anti-adhesive effect reported for poloxamers ${ }^{17}$. Finally, the mucoadhesive effect of this polysaccharide ${ }^{28,29}$ could enhance gel adhesion on peritoneal wall avoiding tissues closing as a barrier component, thus preventing post-surgery adhesion. Alginate and poloxamer also have healing properties widely described. This also attracted us to promote wounds resorption thus reducing possibility of cancer cell implantation ${ }^{30,31}$.

Safety was expected as poloxamers are clinically used in many pharmaceutical formulations, as ophthalmic with cyclosporin eye drops Ikervis $^{\circledR}$, topical with aciclovir ointment Zovirax ${ }^{\circledR}$, or many tablets presentations. But usually there is no medicine with poloxamer authorized for direct peritoneal use. Johnston et al. demonstrated a P407 toxicity dose-dependent relation after repeated IP administration in rats. No variation of hematological parameters rate was observed in rats treated IP with $10 \%$ P 407 for 4 consecutive days compared with control group excepted a slight monocyte increase, whereas rats treated by $30 \%$ P 407 increased almost all parameters evaluated ${ }^{32}$. P407 20 $\%$ ratio in our formulation appear to be a good compromise to avoid systemic toxicity after IP administration. In vivo tolerance of our gel was also assessed. Neither P407 $20 \%$ thermogel nor full thermogel 20/2/1 induced toxicity on blood components, transaminases or main organs such as cerebellum, brain, kidney, small intestine, lung and liver after anatomopathological evaluation. The non-significant slight change in mice body weight which had received P407/P188/alginate thermogel, also reflect the absence of systemic toxicity of our thermogel.

5-FU and Ox dosage in thermogel disclosed that drugs were stable, rejecting the assumption of a potential deleterious effect of the thermogel matrix on the drugs. Invariability of drug retention times in 5-FU/Ox admixture led us to consider that no chemical interaction occurs between both drugs considered sufficiently stable allowing the formulation and the administration of the 5-FU/Ox thermogel in mice. After verification that thermogel did not impaired 5-FU and Ox stability, in vitro toxicity and efficacy of 5-FU-Ox-thermogel were assessed. Obviously, longer lasting delivery systems have been developed earlier and in recent literature ${ }^{33}$. The evaluation of longer lasting systems could also be evaluated in future studies to see whether it would be needed in the context of tumor adhesion post-surgery, knowing that cells will not survive if they do not adhere.

Empty thermogel did not affect cell viability which is consistent with our in vivo toxicity results. Cytotoxicity of 5-Fu/Ox in solution group and thermogel group were similar, proving a non-inferiority of the combined drugs pharmacological effect when entrapped in the thermogel. These results are consistent with the findings of Xie et al. who developed a thermosensitive hydrogel based on polyethylene glycol and $\varepsilon$-caprolactone with P407 nanoparticles of honokiol. P407 showed a very low cytotoxicity on SKOV3 ovarian cells with viability upper than $80 \%$ at $48 \mathrm{~h}$ as compared to untreated cells, independently of its concentration in culture medium. Nanoparticles of P407 also did not 
impact honokiol cytotoxic effect considering cell proliferation curve almost equivalent with honokiol in solution after $48 \mathrm{~h}$ treatment ${ }^{34}$.

Finally, in vivo antitumor efficacy of our 20/2/1 thermogel was evaluated on a peritoneal carcinomatosis murine model. Only the association of both Ox and 5-FU in thermogel was able to significantly decrease the $\mathrm{PCl}$, the number of areas involved, the number of mice with ascites and the mean ascites volume simultaneously. Previously, Jouvin et al. developed an icodextrine based carrier solution to administrate 5-FU and Ox IP and prevent carcinomatosis apparition. In a high risk of growing peritoneal carcinomatosis of colorectal origin murin model, $\mathrm{PCl}$ was significantly inferior in icodextrin 5-FU/Ox group compared to 5-FU/Ox alone, icodextrine alone and control group ${ }^{9}$. We undertook a comparison of $\mathrm{PCl}$ between icodextrine + Ox treatment and thermogel 20/2/1/Ox treatment in our murine model. Results were in favor of the thermogel with a $\mathrm{PCl} 6.4 \pm 1.8$, the only group showing a significant difference compared to control group $(\mathrm{p}<0.01)$ (data not shown).

\section{Conclusion}

In this work, we developed a P407/P188/alginate (20/2/1 \% w/v) non-toxic and biocompatible thermogel for a safety of use. We demonstrated the capability of our thermogel of decreasing CT-26 cells adhesion in vitro. The addition of both $\mathrm{Ox}$ and 5-Fu chemotherapeutic agents in thermogel was as efficient as drugs in solution on cell viability. We therefore proved the in vivo antitumoral efficacy of our drug loaded thermogel on a murine model of carcinomatosis which was superior to the thermogel alone in decreasing of $\mathrm{PCl}$, number areas involved, number of mice with ascites and mean ascites volume. Our poloxamer-based thermogel appears to be a promising drug delivery system to prevent post-surgical adhesion and carcinomatosis formation from primitive colorectal cancer in peritoneal cavity. Further experiments are needed to evaluate the anti-adhesion and anti-cancer efficacy of our thermogel in vivo.

\section{Aknowledgment}

Dr Rachid Kaci perform histological analysis and for helping.

\section{Funding/Financial}

Fondation de l'Avenir for the financial support regarding the project AP-RM-17-023 with INSERM Unit U1275 directed by Pr Marc Pocard. The support is a non-restricted educational grant.

\section{COI/Disclosures}


The authors have no related conflicts of interest to declare Cynthia Pimpie, Pétronille Roy, Al Sabbagh Chantal, Nathalie Mignet, Vincent Boudy: no link of interest

\section{Link of interest declared:}

Marc Pocard : Advisory board or Honoraria : Gamida, Leo-Pharm, Fischer\&Payler, Novartis, Roche, Sanofi, or Grants for research and congress organization: Allergan, AstraZeneca, Bard, Capnomed, Gamida, Fischer@Payler, Integra, Ipsen, Novartis, Rand, Roche, Sanofi, ThermaSolution.

Other authors do not have link of interest to declare.

\section{References}

1. Franko J, Shi Q, Goldman CD, et al. Treatment of colorectal peritoneal carcinomatosis with systemic chemotherapy: A pooled analysis of North Central Cancer Treatment Group phase III trials N9741 and N9841. J Clin Oncol. 2012;30(3):263-267. doi:10.1200/JCO.2011.37.1039

2. Segelman J, Granath F, Holm T, MacHado M, Mahteme H, Martling A. Incidence, prevalence and risk factors for peritoneal carcinomatosis from colorectal cancer. $\mathrm{Br} J$ Surg. 2012;99(5):699-705. doi:10.1002/bjs.8679

3. Honoré C, Goéré $D$, Souadka A, Dumont F, Elias D. Definition of patients presenting a high risk of developing peritoneal carcinomatosis after curative surgery for colorectal cancer: A systematic review. Ann Surg Oncol. 2013;20(1):183-192. doi:10.1245/s10434-012-2473-5

4. Van Santvoort HC, Braam HJ, Spekreijse KR, et al. Peritoneal carcinomatosis in T4 colorectal cancer: Occurrence and risk factors. Ann Surg Oncol. 2014;21(5):1686-1691. doi:10.1245/s10434-013-3461-0

5. Takebayashi $\mathrm{K}$, Murata $\mathrm{S}$, Yamamoto $\mathrm{H}$, et al. Surgery-induced peritoneal cancer cells in patients who have undergone curative gastrectomy for gastric cancer. Ann Surg Oncol. 2014;21(6):1991-1997. doi:10.1245/s10434-014-3525-9

6. Elias $D$, Honoré $C$, Dumont $F$, et al. Results of systematic second-look surgery plus hipec in asymptomatic patients presenting a high risk of developing colorectal peritoneal carcinomatosis. Ann Surg. 2011;254(2):289-293. doi:10.1097/SLA.0b013e31822638f6

7. Klaver CEL, Wisselink DD, Punt CJA, et al. Adjuvant hyperthermic intraperitoneal chemotherapy in patients with locally advanced colon cancer (COLOPEC): a multicentre, openlabel, randomised trial. Lancet Gastroenterol Hepatol. 2019;4(10):761-770. doi:10.1016/S2468-1253(19)30239-0

8. Glehen O, Passot G, Villeneuve L, et al. GASTRICHIP: D2 resection and hyperthermic intraperitoneal chemotherapy in locally advanced gastric carcinoma: A randomized and multicenter phase III study. BMC Cancer. 2014;14(1):1-10. doi:10.1186/1471-2407-14-183 
9. Jouvin I, Najah H, Pimpie C, et al. Reduction of carcinomatosis risk using icodextrin as a carrier solution of intraperitoneal oxaliplatin chemotherapy. Eur J Surg Oncol. 2017;43(6):1088-1094. doi:10.1016/j.ejso.2016.12.009

10. Bodratti AM, Alexandridis P. Formulation of poloxamers for drug delivery. J Funct Biomater. 2018;9(1). doi:10.3390/jfb9010011

11. Roy, P; Mignet, N; Boudy V. Which drug delivery systems to prevent peritoneal carcinomatosis after a cytoreductive surgery of digestives or ovarian carcinoma? Submitted. Published online 2020.

12. Steinleitner A, Lambert H, Kazensky C, Cantor B. Poloxamer 407 as an intraperitoneal barrier material for the prevention of postsurgical adhesion formation and reformation in rodent models for reproductive surgery. Obstet Gynecol. 1991;77(1):48-52.

13. Naim JO, Pulley D, Scanlan K, Hinshaw JR, Lanzafame RJ. Reduction of postoperative adhesions to Marlex mesh using experimental adhesion barriers in rats. J Laparoendosc Surg. 1993;3(2):187-190. doi:10.1089/Ips.1993.3.187

14. Yu CH, Lee JH, Baek HR, Nam H. The effectiveness of poloxamer 407-based new anti-adhesive material in a laminectomy model in rats. Eur Spine J. 2012;21(5):971-979. doi:10.1007/s00586-011-2098-6

15. Kim H-JJ, Kang H, Kim M-KK, and Seung-Su Han, Han SS. The Effects of Barrier Agents in Postoperative Pelvic Adhesion Formation: A Comparative Study of a Temperature-Sensitive Poloxamer-Based Solution/Gel and a Hyaluronic Acid-Based Solution in a Rat Uterine Horn Model. J Laparoendosc Adv Surg Tech. 2018;28(2):134-139. doi:10.1089/lap.2017.0404

16. Cho H, Jammalamadaka U, Tappa K, et al. 3D Printing of Poloxamer 407 Nanogel Discs and Their Applications in Adjuvant Ovarian Cancer Therapy. Mol Pharm. 2019;16(2):552-560. doi:10.1021/acs.molpharmaceut.8b00836

17. Mignet N, Boudy V, Seguin J, Scherman D. Gelling compositions for treating malignant tumors and/or preventing tumor recurrence. 2015;1.

18. Derrien A, Gouard S, Maurel C, et al. Therapeutic Efficacy of Alpha-RIT Using a 213Bi-AntihCD138 Antibody in a Mouse Model of Ovarian Peritoneal Carcinomatosis. Front Med. 2015;2:1-10. doi:10.3389/fmed.2015.00088

19. Glehen O, Kwiatkowski F, Sugarbaker PH, et al. Cytoreductive Surgery Combined with Perioperative Intraperitoneal Chemotherapy for the Management of Peritoneal Carcinomatosis from Colorectal Cancer: A Multi-Institutional Study. J Clin Oncol. 2004;22(16):3284-3292. doi:10.1200/JCO.2004.10.012

20. Lemoine L, Sugarbaker P, Van Der Speeten K. Pathophysiology of colorectal peritoneal carcinomatosis: Role of the peritoneum. World J Gastroenterol. 2016;22(34):7692-7707. 
doi:10.3748/wjg.v22.i34.7692

21. Hansen E, Wolff N, Knuechel R, Ruschoff J, Hofstaedter F, Taeger K. Tumor Cells in Blood Shed From the Surgical Field. Arch Surg. 1995;130(4):387-393.

22. Qiu H ,Guo H, Li D, Hou Y, Kuang T, Ding J. Intravesical hydrogels as drug reservoirs, trends in biotechnology 2020, 38 (6) : 579-583

23. Xia $\mathrm{H}$, Jin $\mathrm{H}$, Cheng $\mathrm{Y}, \mathrm{Xu} \mathrm{Y}$. The Controlled Release and Anti-Inflammatory Activity of a Tetramethylpyrazine-Loaded Thermosensitive Poloxamer Hydrogel. Pharm Res. 2019;36:52. doi:10.1007/s11095-019-2580-0

24. Augst $A D$, Kong $H J$, Mooney DJ. Alginate hydrogels as biomaterials. Macromol Biosci. 2006;6(8):623-633. doi:10.1002/mabi.200600069

25. Koo S, Yang Y, Neu B. Poloxamer 188 reduces normal and phosphatidylserine-exposing erythrocyte adhesion to endothelial cells in dextran solutions. Colloids Surfaces $B$ Biointerfaces. 2013;112:446-451. doi:10.1016/j.colsurfb.2013.07.035

26. Cho WJ, Oh SH, Lee JH. Alginate film as a novel post-surgical tissue adhesion barrier. J Biomater Sci Polym Ed. 2010;21(6-7):701-713. doi:10.1163/156856209X435835

27. Chaturvedi AA, Lomme RM, Hendriks T, van Goor H. Prevention of postsurgical adhesions using an ultrapure alginate-based gel. Br J Surg. 2013;100(7):904-910. doi:10.1002/bjs.9131

28. Goh CH, Heng PWS, Chan LW. Alginates as a useful natural polymer for microencapsulation and therapeutic applications. Carbohydr Polym. 2012;88(1):1-12. doi:10.1016/j.carbpol.2011.11.012

29. Choi HG, Kim CK. Development of omeprazole buccal adhesive tablets with stability enhancement in human saliva. J Control Release. 2000;68(3):397-404. doi:10.1016/S01683659(00)00276-5

30. Lee KY, Mooney DJ. Alginate: Properties and biomedical applications. Prog Polym Sci. 2012;37(1):106-126. doi:10.1016/j.progpolymsci.2011.06.003

31. Percival SL, Mayer D, Kirsner RS, et al. Surfactants: Role in biofilm management and cellular behaviour. Int Wound J. 2019;16(3):753-760. doi:10.1111/iwj.13093

32. Melchior WB, Fahrney D. Ethoxyformylation of Proteins. Reaction of Ethoxyformic Anhydride with $\alpha$-Chymotrypsin, Pepsin, and Pancreatic Ribonuclease at PH 4. Biochemistry. 1970;9(2):251-258. doi:10.1021/bi00804a010

33 Feng X, Li J, Zhang X, Liu T, Ding J, Chen X. Electrospun polymer micro/nanofibers as pharmaceutical repositories for healthcare, J. Controlled Release 2019, 302: 19-41, https://doi.org/10.1016/j.jconrel.2019.03.020

34. Xie $\mathrm{Y}$, Long $\mathrm{Q}, \mathrm{Wu} \mathrm{Q}$, et al. Improving therapeutic effect in ovarian peritoneal carcinomatosis with honokiol nanoparticles in a thermosensitive hydrogel composite. RSC Adv. 
2012;2(20):7759-7771. doi:10.1039/c2ra20612a 